\title{
La calidad de la encuesta
}

\author{
Juan Javier Sánchez Carrión \\ Universidad Complutense de Madrid. Departamento de Sociología IV. \\ 28023 Madrid. Spain
}

\section{Resumen}

El articulo hace una reflexión sobre la bondad/calidad de fa encuesta contemplándola con un triple ojo escrutador: técnico, metodołógico y epistemológico. El ojo récnico busca los posibles errores cometidos en su implementación. El ojo metodológico mira la pertinencia de la lia utilización de la encuesta. Y el ojo epistemológico mira la encuesta después de haber contesrado previamente a una pregunta clave: ¿para qué sirve la investigación?, ¿para explicar la realidad social, para comprender los fenómenos sociales o para transformar el objeto de estudio? Los tres niveles están relacionados en orden jerárquico, de manera que la resptresta que demos al epistemológico influye en el metodológico, y ésre a su vez en el récnico.

Palabras clave: encuesta, calidad, metodologia, errores.

\section{Abstract. The quality of survey}

This article reflects upon the quality of the survey, contemplating with a triply scoutinizing eye, according ro technical, methodological and episcemological perspectives. The technical eye searches for possible errors committed during implementation. The methodological eye views the pertinence of the use of the survey. And the epistemological eyc views the survey after having previously answered a key question: what is the purpose of rescarch? Is it to explain social reality, to understand social phenomenon, or to transform the object of study? The 3 levels are related in hierarchical order, in a way in which the answer we give to the episthemological question influences methodology, and this in turn influences the technical aspect.

Key words: survey, quality, methodology, errors.

\section{Sumario}

1. Contexto problemárico de la encuesta

2. Marco epistemológico

3. Pertinencia metodológica/ epistemológica de la encuesta
4. Exrores en la implementación de la encuesta

Bibliografía 


\section{Contexto problemático de la encuesta}

En este artículo se inicia una reflexión sobre la calidad de la encuesta, abriendo una vía de estudio de esta técnica que la contemple en toda su complejidad. Se trata de ofrecer un esquema tentativo con el que abordar el estudio de la encuesta, que habrá que ir rellenando de contenido en trabajos posteriores - que incluso pueden cuestionar la bondad del esquema--. Así como desde los practicantes de otras técnicas de investigación ha habido una mayor preocupación por las implicaciones teórico-metodológicas de su trabajo - -en nuestro país hay toda una corriente de sociólogos, cuya figura más representativa es Jesús Ibáñez, dinamizadores de la reflexión metodológica en la sociología, tanto cuantitativa como cualitativa-, es mi opinión que desde el Lado de los practicantes de la encuesta se ha dado una mayor autocomplacencia con nuestro trabajo, quizá como resultado de la hegemonía de esta técnica en la investigación social y de la propia dificultad tecnológica que está implícita en su utilización, y cuyo conocimiento prácticamente agota las capacidades de aprendizaje de sus practicantes. Cualquiera que sea la razón, el hecho cierto es que la reflexión metodológica que se realiza desde el lado de los practicantes de la encuesta o es escasa o, cuando se produce, cada vez está más centrada en la discusión de los aspectos que aquí voy a llamar tecnológicos, $y$ que tienen que ver con las condiciones en las que se realiza, pero sin entrar en grandes problemas relativos a su pertinencia o al papel que cumple en la investigación social.

El resultado de la situación descrita en el párrafo anterior es que la reflexión metodológica se ha quedado casi en exclusiva en manos de los filósofos de la ciencia, en general desconocedores de las técnicas que se utilizan en la investigación social, o de personas que no utilizan la encuesta como herramienta regular de su trabajo, y que superada una fase defensiva frente al imperialismo de la encuesta, y "debilitado el enemigo", terminan siendo los únicos que le dan un papel dentro de un esquema general de reflexion sobre el proceso de investigación social.

Como hipótesis subyacente a la realización de este trabajo sostengo que el mayor enemigo de la encuesta es la propia ceguera de quienes la utilizan acríticamente, amparándose en la hegemonía de un modelơ de investigación positivista, en el que la encuesta encontró sus mayores, pero no únicas, glorias. El camino a seguir, como si de un tránsfuga honrado se tratase, es el de recuperar la encuesta como técrica, conociendo bien strs posibilidades y limitaciones (conociendo bien el oficio), para ponerla al servicio de un modelo de investigación que conciba el proceso investigador como construcción y no sólo desvelamiento de la "realidad social».

El título del artículo habla de la calidad de la encuesta, y como ocurre siempre que se piensa en la calidad de algo, lo primero que hay que decir es desde qué perspectiva se procede a la evaluación. En nuestro caso disponemos de un triple ojo escrutador, que nos permite acercarnos a contemplar todos los problemas que afectan al proceso de investigación de la realidad social basado en la 
técnica de la encuesta. Ante los múltiples problemas que acechan al investigador que hace uso de esta técnica, se trata de tener listo un ojo que permita ver la encuesta en su pluridimensionalidad técnica, metodológica y epistemológica: un ojo con componentes técnicos, metodológicos y epistemológicos.

\section{El ojo técnico}

El ojo técnico ha de buscar los posibles errores que se cometen en la implementación de la encuesta. Este ojo no duda de la pertinencia de esta técnica para estudiar el tema en cuestión, pero busca los errores que se hayan podido deslizar en su utilización. Por ejemplo, un requisito de la encuesta es que la muestra sea representativa de la población de la que se extrajo. Si no se cumple este requisito, quizá porque, entre otras circunstancias, haya gente que no responde a la encuesta, lo más probable es que las estimaciones que hagamos estén sesgadas. Igual ocurre si las personas al informar, por ejemplo, de su edad tienden a quitarse años. También en este caso estimaremos sesgadamente la edad de la población a la que trata de representar la muestra. Independientemente de que todas las personas contesten y lo hagan con exactitud, puede ocurrir que también se cometan errores a la hora de analizar los datos obtenidos, quizá debido a que no se utiliza la técnica apropiada, por ejemplo: si se utilizan técnicas de análisis que hacen supuestos no cumplidos por los datos disponibles. En todos estos casos, por motivos distintos, se están cometiendo errores a la hora de utilizar la encuesta: en el primer caso por no disponer de toda la información, en el segundo por tener mala información y en el tercero por analizar mal la información obtenida.

\section{El ojo metodológico}

El ojo metodológico realiza su trabajo antes que el ojo técnico y se pregunta por la adecuación de la encuesta a la dimensión del objeto que estamos estudiando -objeto, por lo demás, definido teóricamente-. Por ejemplo, si queremos conocer los motivos por los que la gente hace determinadas cosas (comprar, votar, etc.), probablemente la encuesta no sea la técnica más adecuada y haya que recurrir a orro tipo de técnicas/metodologías cualitativas (entrevista en profundidad, grupo de discusión, etc.) más pertinentes. Si nuestro objetivo es conocer el comportamiento de los individuos, la técnica de la cncuesta puede ser una técnica idónea. La primera tesponsabilidad del ojo metodológico es decidir si la opción que hacemos es la más acertada para, posteriormente, supervisar que todas las técnicas que se utilizan en la investigación se adecuen al método seleccionado - de la encuesta como técrica a la encuesta como método-. Podría darse el caso de que el ojo técnico certificase la calidad de la muestra que se ha utilizado en una encuesta, mientras que el metodológico cuestionara la pertinencia de haber realizado dicha encuesta.

Alfonso Ortí (19) sistematiza esta pluridimensionalidad del objeto de investigación, que él denomina "niveles y procesos constituyentes de la realidad social", distinguiendo entre diferentes niveles: el nivel de los "hechos", forma- 
do por todo aquello que "acontece y se hace»; el nivel de los «discursos", en el que entraría todo lo que use dice, se expresa o significa en la interacción social"; y el nivel o "reino de las motivaciones", en el que se plantearia el "porqué de la interacción social: su sentido y su intencionalidad of fnalidad, consciente o no consciente". A cada uno de estos niveles de la realidad social le correspondería un modelo metodológico diferente: estadístico (del cual la encuesta sería la técnica paradigmática) para estudiar el nivel de los hechos; modelo linguístico para estudiar los discursos, y modelo heurístico (apoyado en el psicoanálisis y la teoría de la racionalidad) para conocer acerca de las motivaciones.

\section{El ojo epistemológico}

El ojo epistemológico reflexiona sobre el papel de la encutesta y, más en general, de la investigación: ¿para qué sirve la investigación que estamos haciendo? Para ciertas tradiciones de la sociología, basadas en la obra de Durkheim, la investigación ha de servir para explicar la "realidad social", que se presenta conformada por un conjunto de "hechos sociales". Otras tradiciones, con antecedentes en Weber, entienden que con la investigación hemos de ser capaces de comprender el sentido que para los propios individuos tiene su actuación, traducida en forma de «discursos». Por úlrimo cabe una tercera posibilidad, con paternidad en Marx, que se plantea como objetivo del conocimiento la transformación de la realidad objeto de estudio.

A este nivel epistemológico se plantean preguntas sobre la naturaleza del objeto investigado (aquello sobre lo que se investiga), del sujeto investigador (aquel que investiga) y de la relación entre ambos. Si, tal como pienso, el objeto de la investigación es de naturaleza reflexiva - reflexiona sobre lo que se hace con (dice de) él e incluso utiliza esta información para orientar su actuación en el mundo-y el sujero de naturaleza subjetiva (como diría Jesús Ibáñez, sólo se es objetivo para la caza), de identidad parcial a la del objeto investigado y sobredeterminado en sus posibilidades de plantearse preguntas sobre la realidad social por las circunstancias materiales que rodean su existencia, con la investigación lo que se hace no es conocer/reproducir la realidad (el objeto investigado), sino contribuir a su construcción en el propio acto de investigación. Esta labor constructora lleva a enfocar la investigación como un elemento transformador-constructor-definidor de la realidad antes que instrumento que sirve para su explicacion (en términos de porcentaje de varianza explicada de la variable objeto de estudio) o su comprensión (en términos del sentido que tengan sus actos para el propio objeto investigado).

Los tres niveles que acabo de señalar se integran en un todo, el proceso de orientación del investigador en el mundo, con diferentes niveles de responsabilidad - a la manera que el operario de una fábrica es menos responsable que el director, y éste que el dueño de la empresa: el primero pone la técnica, que por definición es aséptica; el segundo pone el método, desde el que se justifica la pertinencia de aquélla, y el tercero define los objetivos, que informan 
todo el proceso anterior-inferior. Cada uno de los niveles - técnico, metodológico y epistemológico - tiene que dar respuesta a una pregunta diferente, ordenada temporal y jerárquicamente: al cómo responde el técnico, al que el metodólogo y al para qué el epistemólogo. La respuesta que demos a cada uno de los niveles determinará la respuesta que demos a los niveles inferiores. Por ejemplo, imaginemos que asumimos el papel transformador de la investigación -frente a una concepción reproductora-. Si esto es así, malamente diremos que hemos cometido un error (de naturaleza técnica) a la hora de utilizar una encuesta para medir mediante una escala alguna característica de los entrevistados, sujeta a transformación-construcción en el mismo acto de investigación; por el contrario habrá que introducir la idea de efecto del instrumento de medida en los resultados, modificando completamente el enfoque de la investigación: ya no se trata de encontrar el instrumento "neutro" que permita conocer la "verdad", sino de conocer nuestro instrumento con el fin de conseguir-construir los efectos deseados, o simplemente de entender los efectos que se producen.

Con este marco de reflexión es con el que debe de abordarse el estudio de la calidad de la encuesta. Entendiendo que hay problemas técnicos, e incluso metodológicos, que son susceptibles de encontrar solución mediante el recurso a un modelo estándar de actuación (más o menos formalizado según el tipo de metodología de que se trate). Pero también entendiendo que hay otros problemas de naturaleza epistemológica para los que no hay una respuesta definitiva, siendo responsabilidad del investigador la toma de partido por una u orra opción, sabiendo que con la respuesta que dé uestará descubriendo su juego, sus cartas y su estrategia", de manera que tal exhibición pueda servir "para potenciar las posibilidades de jugar, él y sus contendientes" (Ibáñez, 1985: 2-3).

\section{Marco epistemológico}

El valor heurístico de cualquier investigación no se puede entender sin una definición previa de cual es la naturaleza del objeto que se pretende investigar -en este caso un objeto sociológico-, del sujeto que investiga y de la relación que existe entre ambos. De manera esquemática abordamos aquí estos problemas. La explicación que se haga de estos aspectos determinará la naturaleza de las técnicas que se utilice y su papel en la investigación social.

Desde una perspectiva epistemológica lo primero que hay que decir es para qué sirve la invescigación -en este caso la encuesta-. La tespuesta ingenua de un hipotético interlocutor sería que para conocer la realidad social. La senciliez de dicha respuesta es más aparente que real, puesto que a tal respuesta enseguida se le superponen nuevas preguntas, en particutar dos:

a) ¿Qué es la realidad social? Dicho técnicamente, ¿cuál es la naturaleza del objeto con el que trabaja el sociólogo?

b) ¿Qué es conocer? Dicho técnicamente, ¿cómo se valida el conocimiento científico? 


\section{Sobre el objeto de la sociología}

El objeto de la sociología es la realidad social, entendida tanto como «realidad real", independientemente de lo que ello sea, como su apariencia - si es que se puede hacer tal diferencia-, en la medida que tanto una como orra influyen en el resto de la realidad o, como diría Beltrán, parodiando la famosa frase, parece que erróneamente atribuida en exclusiva a W.I. Thomas, son "reales en sus efectos" (A.C., 1991: 15); la frase en cuestión dice así: "si los hombres definen las situaciones como reales éstas son reales en sus consecuencias", y según Lamo de Espinosa y otros, 1994, pertenece a Thomas y Swayne, 1928. Esta definición pragmática de lo que es la realidad social (realidad más apariencia) tiene la ventaja de que soslaya el problema ontológico de definir lo que realmente sea y remite directamente a un problema epistemológico, que consiste en explicar cómo la "conocemos".

Definida la realidad social como objeto de la sociología, el paso siguiente es decir cuál es la naturaleza de esta realidad, y si tiene alguna diferencia con las realidades que estudian otros investigadores. Entiendo que lo específico de la realidad social, y que al mismo tiempo la diferencia de orras realidades físiconaturales, es, por un lado, su carácter reflexivo: los hombres (objeto de investigación) reflexionan sobre lo que los investigadores dicen de ellos, cosa que no cabe esperar de las plantas - tal como señala Lamo de Espinosa: "¿qué pasaría si las plantas leyeran libros de botánica?" (A.C. 1990: XI)--; por otro lado, en la realidad social estudiada se encuentran tanto el objeto como el sujeto de la investigación: salvo que se produzca una escisión epistemológica entre ambos, objeto y sujeto comparten identidad.

Decimos que el objeto de la sociología es de naturaleza reflexiva, en dos de los sentidos en los que utilizan este concepto Lamo de Espinosa y otros (1994): los actores pueden saber que se les está investigando, y a partir de este conocimiento modificar sus comportamientos, actitudes, etc.; igualmente, los actores investigados a su vez investigan, con el fin de orientarse en el mundo, para lo cual puede que utilicen las teorías que sobre elios mismos han escrito otros investigadores. Estos aspectos de la reflexividad han sido reconocidos por los sociólogos, bajo concepros tales como los de "profecías que se cumplen a sí mismas" (selffullfilling prophecies, según Merton, 1964) y tenidos en cuenta a la hora de la investigación, recomendando técnicas que se denominan no obstrusivas (unobstrusive measurement), con las que se pretende que el objeto (persona) investigado no sea consciente de esta circunstancia (Webb y otros, 1966).

La importancia de este tipo de reflexividad es que cuestiona la capacidad predictiva de cualquier teoría, modificando el esquema tradicional de predicción, basado en una predicción ajena a la influencia que tal predicción tiene sobre el objeto predicho. Por ejemplo, Lamo y otros ponen un ejemplo de David Riesman, quien mostraba que después de la publicación del libro de Thorstein Veblen sobre la Teoria de la clase ociosa, en 1899, «algunos de los ricos, sientiéndose bajo presión, y a medida que los esloganes del libro se filtraban en la conciencia popular, aceptaron el libro como una guía de lo que no se 
debía hacern (Riesman, 1960: 17, citado por Lamo y otros, 1994: 630). Si Veblen tratase de confirmar la bondad de su teoría, ¿con qué se encontraría? De ser cierto el comentario de Riesman, su teoría habría fallado, podríamos decir del puro éxito que tuvo, en la medida que modificó el comportamiento de las personas investigadas.

La importancia de los párrafos anteriores es enorme, porque cuestiona desde el lado del objeto de la investigación - posteriormente veremos el problema del lado del sujeto- un principio básico de la epistemología positivista, que separa sujeto y objeto de la investigación, atribuyendo al primero el estudio del segundo, de naturaleza distinta a aquél. Frente a este principio de escisión entre sujeto y objeto, la naturaleza teflexiva del objeto sociológico obliga a reconocer la necesidad de incorporar la teoría (el sujeto) como un elemento más que explica el comportamiento de los individuos, uniendo de esta manera sus destinos. Ya no se trata de conocer un objeto exterior, que una razón instrumental intentará manipular hasta la saciedad (a la manera que se manipula la naturaleza), sino de reconocer que, antes que un acto de conocimicnto, lo que la sociología hace es contribuir a la construcción de la realidad social en el mismo acto de estudiarla.

El reconocimiento de esta situación de reflexividad social obliga a darle una solución, puesto que hace insostenible el mantenimiento de los supuestos positivistas de la ciencia, que han informado $e$ informan gran parte de la investigación que se realiza en el ámbito de la sociología. Un intento de alternativa es la creación de lo que Coleman, utilizando uno de los posibles usos del concepto, llama una ciencia social de segundo orden que tome en cuenta la influencia que tiene en la realidad la ciencia social de primer orden (A.C., 1990). Se trata de hacer una sociología que estudie la influencia de la sociología en la realidad. Se parte de la aceptación de que una sociología $S_{1}$, afecta a la realidad:

$$
\mathrm{S}_{1} \longrightarrow \text { Realidad social }
$$

$y$ lo que se propone es una sociología $\$_{2}$ que tenga en cuenta este hecho:

$$
\mathrm{S}_{2} \longrightarrow\left(\mathrm{S}_{1} \longrightarrow \text { Realidad social }\right)
$$

La idea parece interesante, si no fuera porque inmediatamentc se nos ocurre por qué no pensar en una sociología de tercer orden que explique la influencia de la sociología de segundo orden, y así sucesivamente, en una cadena infinita de sociologías. Emilio Lamo y colegas se dan cuenta de este problema al señalar que no hay razones «a priori para interrumpir la reflexividad donde él (Nagel o Coleman, indistintamente) la interrumpe y cabe preguntarse qué ocurriría si $\mathrm{C}_{2}$ (el equivalente a nuestro $\mathrm{S}_{2}$ ) pasara a ser conocido por los actores, dando así lugar a la necesidad de un nuevo conocimiento $\mathrm{C}_{3}$ ral que<smiles></smiles> 
Y por supuesto, esto de nuevo, ad infinitum» (A.C., 1994: 612) 一Jest́s Ibáñez, que ha estudiado en profundidad este problema, hablaría de una cascada transfinita de reflexividades (A.C., 1994: XIV)--. A partir del reconocimiento de la inevitabilidad del recurso a una sociología de órdenes superiores, que dé cuenta de cómo la sociología de primer orden influye en la realidad, los autores citados tienen que admitit la imposibilidad de esta tarea, reconociendo su incapacidad para encontrar una solución al problema, salvo que se produzca un cambio ade las reglas del juego, en este caso las reglas del juego científico" (A.C., 1994: 612) - "cuando algo es necesario e imposible, hay que cambiar las reglas de juegon, diría Jesús Ibáñez (1994: XV) refiriéndose a este mismo problema-. Los autores cifran el cambio de reglas en el propósito de cambiar lo que ellos consideran el enfoque tradicional de la investigación, con el fin de estudiar la influencia de la ciencia social (variable independiente) en la sociedad (variable dependiente), "para indagar cómo esta ciencia (la sociología) es capaz de alterar la sociedad que trata de captar neutralmente y ello, a menudo, sin saberlo. Es decir, que analicemos cuáles son las consecuencias no intencionadas de la acción social investigadora" (A.C., 1994: 632).

En el caso de Coleman, Lamo de Espinosa y colegas han visto muy bien la insostenibilidad de la solución que propone. En su propio caso soy yo el que entiende que no han captado todo el calado que tiene el problema de la reflexividad, como se desprende de la solución teórica (dedicar más o menos atención a tal o cual tema, a sujeto u objeto de la investigación), y no epistemológica, que ellos mismos proponen. Se trata de seguir adelante con su razonamiento constructivista - en definitiva ese es el gran mérito de la teoría de la reflexividad - para aceptar la identidad parcial de sujeto y objeto de la investigación, rompiendo la escisión en la que les ha mantenido toda la tradición científica, tanto natural como social, dominante en las sociedades occidentales desde los tiempos de la Ilustración. Está bien que se reconozca la naturaleza reflexiva del objeto y el papel determinante del sujeto de la investigación, pero todo ello parece insuficiente si no se va hacia el reconocimiento de la identidad parcial existente entre ambos, en un proceso totalizador que tenga como principio de validación no la teoria (de un sujeto sobre un objeto), sino las prácticas sociales (en las que ambos participan). En las siguientes páginas se trata de justificar esta propuesta de solución al problema de la reflexividad.

\section{Sobre el sujeto de la sociología}

Si el objero de la sociología es reflexivo - "sólo es objetivo en la medida que es objetivo para la cazan (Ibánez, 1994: 19)—, el sujeto es subjetivo. Si esto fuera cierto, y yo pienso que lo es, desaparecerfa del vocabulario científico una palabra maldita, junto a todos sus derivados: lo objetivo, la objetividad, tras la que se escudan todo tipo de actos terribles. El sujeto que investiga no sólo influye en (construye) la realidad que dice estudiar, sino que influye en él mismo, en la medida que forma parte de esa misma realidad. Su estatus económico, profesional, etc. puede ser distinto al del objeto investigado, pero no así su natu- 
raleza ontológica. ¿Cómo distinguir al investigador que estudia las condiciones de vida de los trabajadores de estos mismos trabajadores, si no es por su pretensión de situarse fuera (por encima) de los mismos? ¿Acaso sus conclusiones no le van a afectar, para bien o para mal? Desde el momento en que al investigar el investigador se investiga a sí mismo, ¿cómo se puede hablar de objetividad en el proceso del conocimiento?, ¿cómo se puede hablar de objetividad en un contexto en el que ni objeto ni sujeto son objetivos?

Esta idea contraria a la objetividad choca con los planteamientos, tanto de Durkheim como de Weber, que son los autores que han aparecido repetidas veces como paradigmáticos del pensamiento sociológico y sus diferentes orientaciones metodológicas, y que plantean el problema de la objerividad en torno al tema de los valores (juicios de valor) del investigador y su influencia en la investigación. Durkheim acepta la existencia de juicios de valor por parte del investigador, lo que le resta objerividad a la hora de estudiar la realidad social, pero entiende que se trata de una circunstancia pasajera, arribuible a la juventud de la investigación social, si la comparamos con las matemáticas o las ciencias fisico-químicas. Con el tiempo la sociología llegará a este estado de madurez intelectual, en el que se hará posible la primera de sus reglas metodológicas: tratar los hechos sociales como cosas (Dutkheim, 1977). Como muy bien señala Lucien Goldman, el optimismo cartesiano de Durkheim y su poco interés por los problemas de los valores y las ideologías se explican por la utradición optimista de una burguesia - a la que el autor representaba-, que todavía había sido poco sacudida por el desarrollo de un prolecariado que le pudiera oponer su propia visión socialistan (A.C., 1966: 40-41).

En el caso de Weber, las ideologías, los juicios de valor y la presencia del sujeto en la investigación tienen un papel mucho más relevante que en Durkheim - de hecho, el tema de los valores es central para la sociología de Weber-. Para Weber los valores existen, pero solamente intervienen en la investigación en el momento de la elección y la construcción del objeto de investigación; una vez hechas estas elecciones resulta posible estudiarlo de manera objetiva e independiente de los juicios de valor. Por lo tanto, existen múltiples posibilidades, todas válidas, de selección del objeto científico, sin que en la selección esté implícito ningún criterio valorativo. El hecho de que se utilicen metodológicamente unos valores no implica su validez, tan solo nos dicen de su existencia (Beltrán, 1988: 31-32).

Si se da por supuesta la identidad parcial de sujeto y objeto de la investigación (véase Ibáñez, sobre El regreso del sujeto, 1994), tal como se hace en esta exposición, el paso siguiente, y para mi de mayor interés, es determinar la naturaleza de este sujeto de investigación. En primer lugar, se trata de un sujeto reflexivo, en la medida que comparte naturaleza con su objeto de investigación. En segundo lugar, el sujeto de la investigación es un sujeto cuya capacidad de conocer viene determinada, no solo por su capacidad técnica, sino por los limites de su conciencia (Lukács, 1975).

La idea de conciencia posible, asociada a los conceptos de determinismo económico y papel de las clases sociales, me parece central en la investigación 
social, por cuanto muestra, siguiendo a Goldman (1966), que el conocimiento no tiene sus límites en la mayor o menor capacidad técrico-merodológicateórica de los investigadores, sino en sus propias condiciones existenciales - los orígenes de la idea de conciencia posible se encuentran en los tipos ideales de Weber, de quien Lukács fue discípulo aventajado hasta que cambió de orientación teórica-. Se trata de un desarrollo atribuible a Lukács, pero que está en sintonía con todo el pensamiento marxista, resumido en la famosa frase de Marx sobre la influencia que tiene la existencia de los hombres sobre su conciencia, y no a la inversa, y que el autor hizo como crítica a una concepción idealista de la sociedad que anteponia la segunda (la conciencia) a la primera (la existencia). La importancia de la idea de conciencia posible es enorme porque sitúa al sujeto de la investigación en un mundo real, Ileno de limitaciones para el conocimiento que produce (limitaciones propias de sus condiciones existenciales), y no en el limbo de las buenas voluntades, precisamente siempre postuladas pero nunca materializadas, debido a la propia imposibilidad que existe de partida.

Como elemento determinante de esta conciencia se encuentran las condiciones existenciales de los individuos. $Y$ dentro de las condiciones existenciales está la manera como los individuos se ganan la vida, cualquiera que sea la forma de conseguir tan loable objetivo. No se trata de proponer un determinismo económico que explique la vida social única o exclusivamente por los factores económicos, pero sí de atribuirles un lugar privilegiado en la sobredeterminación del comportamiento individual y colectivo. Lo ideológico tiene su lugar, pero subordinado a lo que genéricamente he llamado las condiciones existenciales. Este patrón de relaciones entre lo económico y lo ideológico está muy bien recogido en el prefacio de Marx a la Contribución a la critica de la economía politica. "En la producción social de su existencia, los hombres entran en relaciones determinadas, necesarias, independientes de su voluntad; estas relaciones de producción corresponden a un grado determinado de desarrollo de sus fuerzas productivas materiales. El conjunto de estas relaciones de producción constituyen la estructura económica de la sociedad, la base real, sobre la cual se eleva una supraestructura jurídica y política y a la que corresponden formas sociales determinadas de conciencia. El modo de producción de la vida material condiciona el proceso de vida social, política e intelectual en general. No es la conciencia de los hombres lo que determina la realidad: por el contrario. la realidad social es la que determina su conciencias (A.C., 1970: 37; la cursiva es mía).

\section{Conocimiento praxeológico}

¿Conocer o controlar? Este es el tema central del pensamiento sociológico (y de la ciencia en general). Todo conocimiento es control (puesto que al conocer se participa en la construcción de la realidad que se pretende conocer). Lo que hay es un control positivo (estructural-funcional), que juega con los medios al apostar por un mantenimiento del orden establecido, o un control negativo 
(escuela de Frankfurt), que busca explíciramente el cambio e introduce los fines en el proceso de investigación social. Si a nivel de la calle se acepta la imposibilidad de ser apolítico, dado que siempre se es político, tanto por acción como por omisión, lo mismo se puede decir de la investigación social: se quiera o no, el investigador siempre es un sujeto-subjetivo.

Después de hablar del objeto y del sujeto de la sociología llegamos al punto final de la argumentación, que cierra el marco epistemológico propuesto. Si el objeto de la investigación es reflexivo y el sujeto subjetivo, y ambos forman una unidad indisoluble, ¿cómo se puede evaluar la validez de un conocimiento? Se trata de un problema semejante al de la persona que quiere medir su sombra, cuyo tamaño dependerá de la posición que dicha persona ocupc en relación con la luz que la genera: si se acerca a medirla, o si cambia su posición relativa al sol, la sombra cambia de tamaño. Todo lo que se puede hacer es conocer las leyes de la geometría y del movimiento solar para determinar-producir el tamaño deseado, colocándonos en la posición adecuada con relación al sol.

La esencia reflexiva del proceso investigador (proceso del conocimiento) hace que cuando el individuo proyecta su luz (conocimiento) sobre el objeto que estudia, el objeto se desplaza y cambia de contenido. En estas circunstancias es imposible conocer la esencia del objeto, puesto que tal objeto en ningún momento aparece objetivado (medible) para el investigador. Como muy bien advierte Jesús Ibáfez (1994), se trata de una paradoja, puesto que cuando medimos algo, lo modificamos, lo que convierte a la investigación social en una tarea, a la vez necesaria e imposible. Necesaria porque ni los individuos ni la sociedad pueden renunciar a su propio conocimiento, aunque sea un conocimiento ideológico; imposible, por la identidad entre cl que conoce y lo conocido. En estas circunstancias, ¿cuál es la salida?

Frente a la solución estándar, basada en un racionalismo extremo que sólo acepta la coherencia interna como criterio de verdad, la única salida a la paradoja es ligar el destino del investigador a sus propios conocimientos, no sólo en rérminos del dinero que le reportan, sino del movimiento que provocan en su propia identidad y en las identidades de los demás. Esta solución rompe el estatuto técnico, aséptico, de investigador, distinto al estatuto de investigado, para fundir ambos roles en el único de sujeto en proceso. La práctica será cl único criterio de verdad que se puede utilizar para evaluar la bondad de un saber sobre la realidad social: frente a la explicación positivista o la comprensión fenomenológica, la transformación de la realidad como criterio de validación de la actividad cognoscitiva humana.

Tal como señala Marx en su segunda tesis sobre Feuerbach, "cl problema de si puede atribuirse al pensamiento humano una verdad objetiva no es un problema teórico, sino un problema práctico. Es en la práctica dondc $\mathrm{cl}$ hombre debe demostrar la verdad, es decir, la realidad y el poder, la terrenalidad de su pensamiento. La disputa en torno a la realidad o irrealidad del pensamiento -aislado de la prácrica- es un problema puramente escolásticon (A.C., 1974: 666; cursiva del autor). Y esta práctica transformadora, 
dada la naturaleza social de los hombres, tiene que estar asociada a los intereses del grupo al que los individuos-investigadores pertenecen, lo cual nos lleva a la consideración de su carácter social, que limita -aunque no anula- el relativismo inherente a toda validez basada en criterios pragmáticos - lo que es pragmático para unos no lo es para otros.

Esta reflexión sobre la validación última del conocimiento, de carácter praxiológico, no anula el recurso a la utilización de métodos específicos de investigación, en función de la dimensión de la realidad social que estemos investigando y de los intereses que persigamos. Lo mismo que el hecho de entender la realidad social como una totalidad, en la que las partes se relacionan todas entre sí, haciendo imposible encontrar causas primeras, no evita el recurso a explicaciones causales, en la medida que la vida de las personas así lo exige - si la variable sociedad es la que explica ei comportamiento criminal de algunas personas, miembros a su vez de esa sociedad explicadora, ¿por qué sólo a éstas se las mete en la cárcel, como responsables de sus delitos?-, igualmente, el hecho de que se niegue la existencia de una Verdad (Dios), exterior al sujeto investigador, como criterio de evaluación de la bondad del conocimiento, tampoco evita el recurso a algún criterio para evaluar la verdad de un conocimiento particular. Y este criterio será especifico de la metodologia de investigación que se esté utilizando para obtener-producir ese conocimiento, que a su vez vendrá dererminado por la dimensión del objeto que se esté investigando.

\section{Pertinencia metodológica/epistemológica de la encuesta}

Desde la perspectiva metodológica se produce una reflexión general sobre la pertinencia de la encuesta para responder a los problemas teóricos de la sociología, independientemente de la calidad técnica que pueda tener el trabajo realizado. En la investigación social se produce actualmente un relativo abandono de la encuesta, en la medida que no satisface las expectativas que, injustificadamente, habían depositado en ella muchos sociólogos. De ser la técnica dominante de la sociología, hasta el punto de ser acusada de imperialista y responsable del uempirismo abstracton (Wright Mills, 1971) en el que se mueve gran parte de esta disciplina, pasa a ser una técrica que ha de competir con otras técnicas de carácter cualitativo y participativo, en las que depositan su confianza distintas orientaciones de la sociología. De esta manera, para muchos, la antigua confianza-dependencia se convierte en denostación.

El movimiento hacia lo cualitativo y hacia metodologias participativas tiene su explicación-justificación en la pérdida de legitimidad (llamada crisis) de la sociología occidental: pérdida de legitimidad del paradigma estructural-funcional y, por consiguiente, de su instrumento preferente de investigación, la encuesta. Todo ello producto de la pérdida de legitimidad del modelo de sociedad en el que se inserta la sociología, en tanto que instancia de reflexión cuya función es la de proporcionar la coherencia formal que dicha sociedad necesita para su supervivencia. En este contexto, al tiempo que una parte de los sociólogos vuelven la vista hacia metodologías cualitativas y praxeológicas, 
algunos de los que estamos en el campo cuantitativo debemos orientarnos hacia un proceso de "ajornamiento", consistente en integrar, en la medida de lo posible, las críticas que desde las otras perspectivas de investigación se hace de nuestro trabajo, relativas a las limitaciones del método de la encuesta, y en conseguir un mayor refinamiento de nuestros propios instrumentos de investigación. Después de un primer momento de enfrentamiento nítido entre las diferentes perspectivas, lógico en el proceso de consolidación de una de ellas (la cualitativa) -por el momento, la praxeológica deberá esperar-y de aceptación de la pérdida de su papel hegemónico por parte de la otra (la cuantitativa), el resultado del conflicto puede tener un marcado carácter positivo, en la medida que cada metodología se beneficie de los aspectos positivos de la otra, estableciéndose entre ellas una situación de complementariedad.

En todo este contexto problemático que acabo de presentar, en el momento actual la encuesta se ve enfrentada a una serie de dilemas, a los que responde con mayor o menor éxito, sola o combinando su actuación con otras metodologías, y de los cuales yo ofrezco una relación, sin ánimo de exhaustividad:

- Explicación causas-comprensión sentido. Como consecuencia de la presión cualitativista, que acentúa el interés por lo subjetivo y la construcción del sentido que a sus acciones atribuyen los individuos, se produce toda una orientación hacia el estudio de los procesos comunicativos que se dan en la encuesta, dando un papel privilegiado al hasta entonces entrevistado-fronrón-caja negra de las entrevistas - descubrimiento de la psicología cognitiva (Bradburn y Danis, 1984; Tanur y Fienberg, 1992).

- Sincrónico-diacrónico. Tampoco es casualidad la imporrancia que adquiere el estudio del cambio, y el desarrollo que toma la creación de las bases de datos, tipo barómetro, y todas las técnicas de análisis longitudinal, tipo análisis de series temporales o análisis de eventos (event analysis), coincidiendo con una sociedad que se encuentra sumida en algo más que un proceso de cambio lineal. La recuperación de la historia como atalaya desde la que contemplar y comprender la vida social necesariamente se refleja en la preocupación que tienen los metodólogos en introducir la variable tiempo en los modelos estadísticos.

- Colectivismo-individualismo metodológico. ¿Y qué decir de la importancia que adquiere el estudio del contexto en el comportamiento individual, superando la antinomia individuo-sociedad? A partír de los años ochenta se desarrolla toda una corriente de estudio denominada análisis a múltiples niveles (multi-level analysis), que tiene como objetivo desarrollar tecnologías (basadas en el modelo de la regresión) que permican cuantificar la influencia de la sociedad (familia, escuela, etc.) en aspectos propios de los individuos (rendimiento escolar, voto, etc.) (Goldstein, 1987; Paterson y Goldstein, 1991).

- Realismo-constructivismo. ¿Y acaso en la orientación hacia un tipo de investigación no obstrusiva no está latente la preocupación por el carácter refle- 
xivo del objeto-individuo de la sociología y las consecuencias no intencionadas de la acción? (Webb, 1966).

- El problema de la medida. No menor importancia tiene toda la reflexión que hacen autores como, por ejemplo, Ray Pawson tratando de resolver el enfrentamiento entre empiristas y teoricistas, en base a una revisión del concepto central de toda metodología: la medida (Pawson, 1989).

- Lógica de la contrastación-lógica del descubrimiento. Por último, y sin ánimo de agotar la elucidación de las vías de interpenetración entre perspectivas y los frentes que tiene abiertos la encuesta, hay que citar la importancia de los análisis exploratorios (frente a los confirmatorios), en una sociología que ni después de hacer bueno el desideratum mertoniano de buscar teorías de alcance medio encuentra ámbitos de seguridad; en este contexto adquiere especial relevancia la técnica del análisis exploratorio de los datos (EDA) (Tukey, 1977).

\section{Errores en la implementación de la encuesta}

Desde el lado de los practicantes de las técnicas cuantitativas, una vez que se acepta su pertinencia como técnica más adecuada para investigar determinados aspectos de la realidad social, se produce una reflexión sobre la calidad de la encuesta, con una gran preocupación por los errores que se cometen en su utilización. Libros como los de Groves (1989), Survey Errors and Survey Costs; Biemer y otros (1991), Measurements Errors in Surveys; o los monográficos de las revistas Sociological Methods and Research - dirigido por Alwin (1991), y titulado Research on Survey Quality-y Journal of Official Statistics (1992) - organizado por Biemer y Lyeberg-; así como los numerosos artículos que aparecen en las revistas especializadas, dedicados a la calidad de la encuesta; o la también numerosa presentación de ponencias en congresos y jornadas de metodología, relacionadas con este tema, serían la expresión del interés, creciente, que despierta en la comunidad profesional la calidad asociada a la técnica de la encuesta - bien es cierto que en este movimiento, cada vez más tecnificado, los sociólogos vamos cayendo, para dejar paso a los estadísticos de encuestas, y a los especialistas en psicometría o en econometría.

Desde un punto de vista conceptual, la investigación sobre los errores se suele dividir en tres partes diferenciadas. Por un lado, los investigadores son conscientes de que en toda encuesta se cometen errores atribuibles a que no se obtiene toda la información necesaria (por ejemplo, para saber lo que piensa una Población sólo se pregunta a una Muestra). Siguiendo a Groves (1989), en quien está parcialmente basada esta tipología, a este tipo de errores le voy a Ilamar de "no observación», puesto que ésta es la razón de que aparezcan. Por otro lado, aún cuando obtenemos información de las personas entrevistadas, es muy probable que contenga errores de medida (por ejemplo, el entrevistado no siempre dice lo que piensa, el instrumento urilizado influye en la respuesta, etc.); por lo tanto, este segundo bloque de errores recibe el nombre de erro- 
res de medición. Por último, aún en el supuesto de que no haya errores de medición, el tratamiento informático estadístico que se hace de los datos, con el fin de sacar las conclusiones, puede originar nuevos errores, a los que voy a llamar de cálculo.

\section{Errores de no observación}

Entre los errores de no observación los investigadores estudian los problemas atribuibles a que algunos miembros de la población estudiada no están incluidos en el marco muestral (por ejemplo, población que no tiene teléfono en un estudio de la población general), mientras que otros aparecen dos o más veces (por ejemplo, todos aquellos que tienen varias líneas de teléfono). Este tipo de errores reciben el nombre de errores de cobertura. La solución al problema pasa por un estudio detallado del marco muestral que se utiliza en la investigación, con el fin de detectar este tipo de errores de cobertura, y de adaptarlo, si es posible, a las condiciones de la población investigada, o, alternativamente, redefinir la población en términos del marco que se utiliza.

También se estudia el problema que surge cuando algunos miembros de la muestra no son entrevistados, bien porque no es posible contactarlos o porque rechazan participar, total o parcialmente, en la encuesta. Cuando se produce esta circunstancia estamos en presencia de un error de no respuesta. Dependiendo de que la no respuesta lo sea a la totalidad o a una parte (pregunta) del cuestionario, hablamos de no respuesta total o parcial, respectivamente. La diferencia es pertinente por las consecuencias diferenciadas de cada tipo y por el distinto tratamiento que reciben. Si las personas que no contestan son una submuestra aleatoria de la muestra seleccionada - dicho de otra manera: son iguales a las que contestan-, la no respuesta tan sólo disminuye el tamaño de la muestra, lo que incrementa el error variable de muestreo - también llamado simplemente error de muestreo, según infra-. En el caso de que las personas que no contestan sean distintas a las entrevistadas, al problema de la disminución del tamaño de la muestra - evitable si se sustituyen a las personas que no contestan por otras "contestadoras"- se añade el del sesgo que se produce en las estimaciones de los parámetros poblacionales, que aparecerán sobre o infraestimados.

Por último, el hecho de que se urilice sólo una parte de personas (la muestra) para conocer lo que piensan todas ellas (la población) añade un tercer tipo de error, Ilamado error de muestreo: si queremos saber lo que piensan muchas personas a partir del conocimiento que tenemos de lo que piensan unas pocas, es comprensible que cometamos algún error en la extrapolación. Por definición, la muestra es más pequeña que la población, y de esta circunstancia se deriva que al utilizarla se cometa un tipo de error llamado error variable de muestreo -el error muestral que se suele calcular en las encuestas-; si además la muestra no es representativa, quizá debido a la presencia de los dos tipos de errores previamente mencionados, hay que añadirle un nuevo error, el sesgo, llamado error fijo de muestreo. La suma de ambos errores determina el llamado 
error medio cuadrático o error total de muestreo (sobre los errores de muestreo véase Sánchez Carrión, 1995).

Errores de no observación $\left\{\begin{array}{l}\text { Errores de cobertura } \\ \text { Errores de no respuesta } \\ \text { Errores de muestreo }\end{array}\right.$

\section{Errores de medición}

Mayor interés que los errores de no observación reciben los errores de medición: las diferencias que se dan entre los (supuestos) verdaderos atributos de los entrevistados y los resultados que se obtienen al tratar de conocerlos utilizando la técnica de la encuesta. A este nivel se plantean dos enfoques de investigación distintos, dependiendo de los supuestos epistemológicos que haga cada investigador. Un enfoque posible, que es el que subyace a la práctica regular de la encuesta, parte de unos supuestos epistemológicos que atribuyen realidad objetiva a las características de los individuos y que confia en la neutralidad del instrumento técnico utilizado, capaz así de captar esa realidad. Desde esta perspecriva, los errores de la encuesta son verdaderos errores, y se producen cuando no mide lo que hay que medir: el individuo tiene un valor de 8,0 en una variable (por ejemplo, la valoración que tiene de su profesor de estadísrica) y la encuesta nos da un 7,7. Puesto que el 8 tiene un valor de realidad, si la encuesta llega a la obtención de otro número, ello es debido a que hemos cometido algún error. A este enfoque se le puede poner el nombre de realista, $y$ es uno de los pilares del mérodo positivista de investigación.

Desde un enfoque distinto al del párrafo anterior, al que se puede llamar constructivista, se empieza por dudar de que haya habido un error en la encuesta, puesto que no está claro que el individuo tenga realmente un valor de 8 , independientemente de cuál sea el proceso de medición. Frente a la idea de una realidad objeciva, medible con las herramientas del investigador (en este caso, la encuesta), se plantea la idea de una realidad socialmente construida, en este caso por el investigador y las herramientas que utiliza, que actuarían como elementos que introducen información en la sociedad y no como elementos que sirven para obtenerla. Desde esta perspectiva, los errores de las técnicas se reconvierten en efectos de las técnicas, queridos o no queridos por el investigador.

La consecuencia de que se adopte una perspectiva realista o constructivista - con todas las matizaciones que quepa hacer en el continuun definido por esas dos perspectivas, que actúan como polos- es fundamental, puesto que determina el curso que debe seguir la investigación por encuesta, situada en una encrucijada, por la pretensión que tienen muchos de sus practicantes de seguir manteniendo la ficción de que es posible desarrollar un instrumento neutro, con el que descubrir la verdad. Pensemos sólo en un problema como el de dar o no dar incentivos a los entrevistados: a partir de una concepción realista se plantea toda una polémica en torno a la pertinencia de esta prácti$\mathrm{ca}$, alegando su posible efecto distorsionador sobre las respuestas que dan los entrevistados. Es cierto que si se paga a los entrevistados, este hecho determina lo que contestan, pero no menos cierto es que si no se les paga, también 
este hecho influye en sus respuestas, puesto que ninguna de las dos situaciones es la "natural» $y$ "neutra», frente a la cual la otra introduciría distorsión en las respuestas.

Mí opinión es que, no sólo los dos supuestos sobre los que se trata de sustentar la técnica de la encuesta, realismo social y neutralidad de la técnica, no están justificados, sino que además están entorpeciendo el desarrollo de la encuesta - a la manera que el modelo de sociedad capitalista entorpece actualmente el desarrollo de la humanidad-. Lo que para muchos investigadores son efectos no queridos (por ejemplo, por dar incentivos o utilizar una u otra formulación de las preguntas de los cuestionarios), y por lo tanto efectos perversos de la encuesta, que hay que eliminar para que esta técnica alcance su plenitud -algo que se desvela como imposible-, en realidad son parte consustancial a la encuesta, y sólo aceptando esta realidad, que libere a la técnica de la carga metodológica y epistemológica positivista que la acompaña, podrá realmente progresar.

Lo que unos (realistas) calificarán como un error de la técnica, que infravalora la realidad, otros (constructivistas) lo calificarán como un efecto de la técnica, que tiende a infravalorar-sobrevalorar resuitados obtenidos con otra técnica. Para los primeros habrá un absoluto (la verdad), que actuará de estándar de comparación - en este planteamiento se mueve toda la teoría clásica de la medida-, mientras que los segundos se plantearán problemas de relatividad - lo cual no impide el consenso en unos procedimientos de trabajo ajustados a unos estándar de calidad-. En lo sustantivo hay coincidencia en descomponer los elementos de la encuesta, con el fin de estudiar los errores/efectos que se producen en torno de ellos: se podrá tener diferencias a la hora de valorar la influencia del método de recogida de la información (entrevistas cara a cara o telefónica) en los resultados, pero está claro que hay que considerar este factor si queremos comprender la información que se ha obtenido en una encuesta.

Según Groves (1987), los errores pueden aparecer debido a: a) la influencia que tiene el entrevistador sobre el entrevistado (su forma de actuar, de vestir, etc.), b) la naturaleza de las preguntas del cuestionario (su formulación, el orden en el que aparecen las alternativas de respuesta, etc.), c) la actuación del entrevistado a la hora de contestar a las preguntas que se le formulan (quizá no tiene interés en decirnos la verdad, trata de agradar, etc.) y $d$ ) a la influencia del modo de recoger la información de las personas estudiadas (cuestionario postal, entrevista telefónica, etc.).

Debido a que es más fácil estudiar este tipo de errores que los de no observación (que por definición son difíciles de observar), la literatura está llena de estudios en los que se analiza la influencia de todos estos factores que acabamos de enumerar, mientras que son menores los estudios del primer tipo.

$$
\text { Errores de medición }\left\{\begin{array}{l}
\text { Errores en torno al entrevistado } \\
\text { Errores en tomo al entrevistador } \\
\text { Errores en como al instrumento } \\
\text { Errores en tomo al método }
\end{array}\right.
$$




\section{Errores de postmedición}

A estos dos tipos de errores aún cabría añadir un tercero, verdaderamente error y no efecto, relacionado con el procesamiento de la información que se obriene en la investigación y su posterior análisis. Ya no se trata de que los datos sean parciales (referidos a una muestra) e inseguros (con errores de observación), sino que ahora hay que añadir el hecho de que todos los datos obtenidos tienen que ser tratados informática y estadísticamente, con el fin de sacar conclusiones. El procesamiento corresponde a la fase que va desde el momento en que se han cumplimentado los cuestionarios (soporte de recogida de la información) hasta que se realizan los análisis definitivos (regresión, tablas de contingencia, etc.). Se trata de una etapa muy delicada, dado que en las encuestas se trabaja con miles de observaciones ( $n$ casos por $p$ variables) $-\mathrm{y}$ a veces, millones-. En estas circunstancias la grabación de esta información y su edición (editing) es todo un proceso industrial, sometido a optimización y control. A tal fin existen procedimientos estándar de grabación y validación de los datos -especialmente en los grandes centros estadísticos- que sirven para realizar todo este proceso (véase Villán Criado y Bravo Cabria, 1990). Básicamente, esta etapa de procesamiento comprende la grabación, la depuración de errores y la transformación de los datos.

En la grabación se trata de garantizar que la matriz de datos con la que se realizarán los análisis refleja fielmente los resultados obtenidos con los cuestionarios. La depuración se suele hacer para detectar códigos inválidos (por ejemplo, la pregunta tiene dos alternativas de respuesta, 1 y 2 , y en la matriz aparece un 4) y para detectar inconsistencias en las respuestas de los entrevistados (o en la grabación de los datos): si la persona dice tener diez años (variable edad) no puede contestar que tiene carnet de conducir (variable permiso). La transformación pretende poner los datos "guapos" para el análisis: por ejemplo, tenemos la variable $e d a d$ en años e interesa utilizarla agrupando las edades en intervalos; o, quizá, en diferentes preguntas se indaga sobre la posesión de diferentes objetos y queremos construir un indice de posesión de objetos, que nos diga en una sola variable el número total de objetos poseídos por cada individuo.

\section{Errores de postmedición $\left\{\begin{array}{l}\text { Errores de procesamiento } \\ \text { Errores de análisis }\end{array}\right.$}

Después de que los datos están listos, hay que analizarlos. También a este nivel se presentan múltiples posibilidades de cometer errores. Si se trata de datos muestrales, el primero de ellos es su incumplimiento de los supuestos especificados en el modelo de análisis utilizado (quizá la muestra es pequeña, la población no es normal, y el test es paramétrico). A nivel del análisis también se pueden cometer errores si el nivel de medición de los datos no se corresponde con las exigencias de la técnica que se va a emplear (por ejemplo, los datos son ordinales y la técnica utilizada exige información interval). Los orde- 
nadores y la utilización de paquetes informático-estadisticos en la investigación social han facilitado el análisis que se hace de la información obtenida, en la medida que simplifican totalmente cálculos que antiguamente exigían un esfuerzo muy considerable, pero esa misma facilidad de obtener resultados instantáneamente y sin apenas esfuerzo también ha creado las condiciones para que investigadores inexpertos realicen toda suerte de análisis estadísticos con una ignorancia casi absoluta de lo que están haciendo.

\section{Bibliografía}

AI.WIN, D. (1991). «Research on survey data quality". Sociological Methods and Research, vol. $20,1: 3-30$.

Bitrrán, Miguel (1988). Ciencia y Sociologiá. Madrid: Centro de Investigaciones Sociológicas.

- (1991), La Realidad Social. Madrid: Tecnos.

Biemer, Paul; Groves, Robert; LyberG, Lars; Mathiowetz, Nancy; Sudman; Seymour (1991). Measurement Errors in Surveys. Nueva York: John Wiley \& Sons.

BRADBURN, Norman M.; DANis, Catalina (1984). "Potential contributions of cognitive research to survey quescionnaire designm. En Jabinc, T. y otros. Cognitive Aspects of Survey Methodology. Building a Bridge between Disciplines. Washington: National Academy Press.

Col.EMAn, James S. (1990). Foundations of Social Theory. Boston: Harvard University Press.

DurkHeIm, Emile (1977). Las Reglas del Metodo Sociológico. Buenos Aires: Editorial la Pléyade.

Goldman, Lucien (1966). Sciences Humaines et Philosophie. Pazis: Editions Gonthier.

- (1975). Marxismo y Ciencias Humanas. Buenos Aires: Amorrortu.

Goldstein, Harvey (1987). Multilevel Models in Educational and Social Research. Londres: Griffin.

Groves, R.M. (1987). "Research on survey data quality». Public Opinion Quarterly, vol. 51, 3: 156-172.

- (1989). Survey Errors and Survey Costs. Nueva York: John Wilcy \& Sons.

IBÁÑEZ, Jesús (1985). Del Algoritmo al Sujeto. Perspectivas de la Investigación Social. Madrid: Siglo XXI.

- (1994). El Regreso del Sujeto. Madrid: Siglo XXI.

Journal of Official Statistics (1992). Volumen dedicado a la calidad de la encuesta. Vol. 8, núm. 1 y 3. Publicado por el Instituto de Estadística Sueco.

Lamo de Espinosa, Emilio (1990). La Sociedad Reflexiva. Sujeto y Objeto de Conocimiento Sociológico. Madrid: Centro de Invescigaciones Sociológicas.

Lamo de Espinosa, Emilio; GoNZÁlez García, José María; Torres Al.bero, Cristóbal (1994). La Sociologla del Conocimiento y de la Ciencia. Madrid: Alianza Universidad Texros.

LuKÁCS, Georg (1975). Historia y Conciencia de Clase. Barcelona: Grijalbo.

MARX, Carlos (1970). Contribución a la Crítica de la Economía Politica. Madrid: Alberto Corazón Editor.

- (1974). La Ideologia Alemana. Barcelona: Grijalbo. 
Merton, Robert K. (1964). Teoria y Estructura Sociales. México: Fondo de Cultura Económica.

MiLls, C. Wright (196I). La Imaginación Sociológica. México: Fondo de Culfura Económica.

OrTf, Alfonso. "El proceso de investigación de la conducta como proceso inregral: complementariedad de las técnicas cuantitativas y de las prácticas cualitarivas en el análisis de las drogodependencias". En Las drogodependencias: perspectivas sociologicas actuales. Madrid: Colegio Nacional de Doctores y Licenciados de Ciencias Polfticas y Sociología.

Paterson, L.; GOlSTEIN, H. (1991). "New staristical methods for analysing social structures: an introduction to multilevel models". British Educational Research Journal 17: 387-394.

PAWSON, Ray (1989). A Measure for Measure. A Manifesto for Empirical Sociology. Londres: Routledge.

RJESMAN, David (1960), Thorstein Veblen: a Critical Interpretation. Nueva York: Scribner.

SANCHEZ CARRION, Juan Javier (1995). Manual de Análisis de los Datos. Madrid: Alianza Universidad Textos.

TANUR, Judith; FiENBERG, Stephan E. (1992). "Cognitive aspects of survey: yesterday, today and tomortowm, Jornal of Official Stadistics, vol. 8, 1: 5-17.

TUKEY, J. (1977). Exploratory Data Analysis. Reading, Mass: Addison-Wesley.

VILIÁN CRIADO, IIdefonso; BRAVO, Ma Soledad (1990). Procedimiento de Depuración de Datos Estadisticos. Cuaderno núm. 20. Vitoria: Instituto Vasco de Estadística.

WEBB, Eugene; CAMPBElL, Donald T.; SCHWARTZ, Richard D.; SECRFST, Lee (1966). Unobstrusive Measures. Chicago: Rand McNally.

Weber, Max (1979). Economia y Sociedad. México: Fondo de Cultura Económica. 